\title{
Étude des difficultés conceptuelles des élèves marocains sur la formation d'image à travers une lentille convergente et la vision
}

\section{Study of Marrocan pupils' Conceptual difficulties about Image Formation by Lenses and the vision}

\author{
Ahmed Sidi Cheikh ${ }^{1 *}$, Khalid Ahaji ${ }^{2}$, and Ahmed Belmoudene ${ }^{1}$ \\ ${ }^{1}$ Laboratoire Apprentissage Cognition et Technologies éducatives, FSE, Université Mohamed V de \\ Rabat, Maroc. \\ ${ }^{2}$ Centre d'Orientation et de Planification de l'Education, Rabat, Maroc.
}

\begin{abstract}
Résumé. Cette recherche vise à explorer les difficultés conceptuelles lié à la formation d'image optique et la vision, chez les élèves de la deuxième année secondaire collégial; avant et après l'enseignement ordinaire de l'optique géométrique. L'échantillon de cette étude est composé de 52 élèves, dont 22 du groupe expérimental et 30 du groupe du groupe control. Les données ont été collectes à travers un questionnaire (pré-test et posttest). Cet article a exploré quelques conceptions alternatives chez les élèves, à propos du rôle de la lentille dans la formation d'image et le processus de vision. Nous examinons, ensuite, l'effet d'une activité d'apprentissage basée sur les nouvelles technologies, sur l'évolution conceptuelle des élèves. L'analyse statistique de résultats a montré qu'il y a une différence statiquement significative, entre le résultat du groupe expérimental et le résultat du groupe contrôle. Le groupe expérimental a enregistré une amélioration significative, plus de $49 \%$.
\end{abstract}

\begin{abstract}
This research aims to explore the conceptual difficulties related to image formation and vision, at the students in the second year college; before and after instruction ordinary in geometrical optics. The sample for this study was composed of 52 students, which 22 from the experimental group and 30 from the control group. The data were collected through a questionnaire (pre-test and post-test). This article explored some alternative conceptions at the students, about the image formation by lenses and process of vision. We then examine the effect of a learning activity based on new technologies (computer simulations), on the of students' conceptual change. The analysis Statistical of the results showed that there was a difference
\end{abstract}

*Corresponding author: ahmed.baba29@yahoo.fr 
statically significant between the results of the experimental group and the control group. The experimental a significant increase of more than $49 \%$.

\section{INTRODUCTION ET PROBLEMATIQUE}

Les élèves ont des conceptions erronées relatives à différents concepts en optique géométrique. Dans différentes niveau scolaire les élèves possèdent des conceptions erronées bien ancrées sur la lumière, la vision et la formation d'image à travers une lentille convergente, le mécanisme de la vision $[1,2,3,4]$. D'autres chercheures, ont révélé que les élèves formalisent des conceptualisations "holistique", sur la notion d'image formée à travers une lentille convergente, avant une séance d'enseignement d'optique géométrique [2]. Selon ces élèves, la présence d'écran est nécessaire pour la formation d'image à travers une lentille convergente. Ainsi, autres chercheures, ont mis en évidence que les élèves ont des difficultés conceptuelles à propos de l'optique géométrique ; ils ont cité, entre outre, que l'image d'un objet lumineux à travers une lentille convergente persiste après suppression de la lentille [5]. Dans une autre recherche, [1] a soulevé que les conceptions des élèves sur le processus de formation d'image sont incomplète; certains d'entre eux s'inscrivent en infraction avec le savoir scientifique. De plus, d'autres recherches ont soulevé, entre outre, des difficultés conceptuelles sur la propagation de la lumière, processus de la vision, comme elles sont identifiées dans la bibliographie [6]. De plus, plusieurs autres chercheures, ont montré que les élèves acquièrent des connaissances sur les phénomènes scientifiques et, les différents concepts scientifiques, à travers leurs expériences vécus; ainsi qu'avec l'interaction d'environnement qui les entoure $[7,8,9,10]$. [8,11] ont mentionnées que ces conceptions, comme étant stable, robustes et souvent résistifs aux changements ; même après l'apprentissage. La mise en évidence de ces conceptions, permet à l'enseignant de choisir les stratégies et, le contenus adéquates pour faire évoluer les conceptions des élèves et de les remplacer par des conceptions qui se rapprochent de celles admise par la communauté scientifique [12]. En fait, si les enseignants ne tiennent pas compte de ces conceptions erronées des élèves, des vestiges de ces conceptions peuvent apparaître plus tard comme un obstacle à l'apprentissage des concepts scientifiques [2,13]. Pour cette raison, nombreux chercheures, ont indiqué que les conceptions erronées des élèves doivent être le point de départ, afin de faire procéder l'enseignement des phénomènes et concepts scientifiques $[8,7]$.

De nombreuses discussions théoriques ont été menées sur la façon dont l'apprenant acquiert les connaissances, ainsi, le rôle de connaissances erronées dans l'acquisition de nouvelles connaissances scientifiques. La question majeure qui a été posée est la suivante : dans quelle mesure les conceptions erronées des élèves restructurées (ou évoluées) au cours de l'apprentissage ? Des chercheures, ont décrit l'organisation des connaissances en termes de schémas et proposent trois types d'apprentissage : l'accrétion, la structuration et le réglage. L'accrétion est l'ajout de nouvelles connaissances à la mémoire existante [2]. La structuration implique la formation de nouvelles structures ou schémas conceptuels. Le réglage est l'ajustement des connaissances à une tache spécifique généralement par la pratique. L'accrétion est la forme d'apprentissage la plus courante ; la structuration est beaucoup moins fréquente et nécessite des efforts considérables; le réglage est la forme d'apprentissage la plus lente et représente la performance des experts. D'une autre part, Piaget considère qu'il y a deux processus principaux pour la régulation de connaissances : l'assimilation et l'accommodation. L'assimilation est un processus mental responsable à partir de l'environnement et de le mettre dans une nouvelle structure cognitive de l'individu ; l'accommodation vise à réajuster le processus mental en fonction de transformation subies [12]. 
Dans ces dernières années, plusieurs modèles ont été émergés quant à la structure des conceptions erronées. Nous citons, parmi eux, les modèles de remplacement des conceptions inspirées par les contributions qui ont été menées par, Piaget (1952), Kuhn (1962), et de conceptions fondamentaux d'accommodation de révolution scientifiques et de dissonance cognitives qu'ils défendirent (respectivement). Les premières propositions complètes de modèle du changement conceptuel furent basées sur l'idée fondamentale que les conceptions des élèves, pour évoluer, devrait d'abord être, en quelque sorte, mise en échec [8]. Nous avons basé, sur, ce modèle pour faire une conception d'une activité d'apprentissage qui favorise l'évolution et le remplacement des conceptions erronées par des conceptions plus scientifiques.

Le but de la présente recherche est de révéler les conceptions erronées chez les élèves marocains de la deuxième année du secondaire collégial à propos de l'optique géométrique. De plus, de montrer que l'utilisation de nouvelles technologies dans l'enseignement de sciences physiques, et notamment l'optique géométrique à un impact positif sur l'évolution des conceptions des élèves. De même, nous confirmons que l'enseignement traditionnel est inefficace pour faire, le changement conceptuel, chez les élèves et il n'aide pas les élèves à faire la dissonance cognitive, dans le but de faire ce changement conceptuel. Par conséquent, dans cette recherche, nous tenterons de répondre aux questions suivantes :

1. Quelles sont les conceptions erronées chez les élèves de la deuxième année du secondaire collégial, avant et après une séance d'enseignement, la formation d'image et le mécanisme de vision ?

2. La méthode d'enseignement traditionnel, est-elle efficace pour enseigner les concepts en optique géométrique?

3. Quel est l'apport de l'utilisation du logiciel OptgGeo dans l'évolution de conceptions des élèves et de la meilleure compréhension de concepts en optique géométrique?

\section{METHODOLOGIE}

Cette recherche s'est portée dans deux perspectives. Tout d'abord dans une perspective descriptive, nous avons identifiées les conceptions erronées des élèves sur la lumière, la formation d'image et le mécanisme de vision. Avant et après qu'ils reçoivent un enseignement ordinaire (formelle) à l'école. D'une autre part, nous avons fait une intervention à travers une activité d'apprentissage basé sur les nouvelles technologies (logiciel de simulation), dans le but de déstabiliser les conceptions erronées qui ont été révélées. En fin, nous avons fait une analyse statistique de résultats des deux groupes (expérimental et contrôle).

\subsection{L'échantillon}

52 élèves de la deuxième année du secondaire collégial d'un établissement public situé à Rabat (Maroc) ont participé à cette recherche. Les élèves ont été réparties en deux groupe (expérimental et contrôle), le groupe expérimental est composée de 22 élèves (14 garçons, 8 filles), le groupe témoin est composée de 30 élèves (14 garçons, 16 filles) ; l'âge moyen des élèves était 12 ans. 


\subsection{Instruments de collecte de données}

Nous avons utilisé l'administrateur pré-test et posttest, comme instrument pour le dépistage des conceptions erronées et d'analyser les compréhensions conceptuelles des élèves. Cet administrateur est composé de 15 questions qui couvrent les différents concepts visés, dans le programme de l'optique géométrique enseignées en deuxième année du secondaire collégial (propagation rectiligne de la lumière, formation d'image et le mécanisme de la vision).Deux mois après, le pré-test, les élèves du groupe expérimental ont été invité aux interactions de tutelle qui vise à la déstabilisation de leur conceptions erronées, tandis que les élèves du groupe témoin ont suivi l'enseignement de l'optique géométrique dans le classe normale(méthode ordinaire) et dans le même cadre des horaires scolaires.

\subsection{Procédure}

La présente recherche a été réalisée au cours du deuxième semestre de l'année scolaire 2019/2020 dans une école publique à Rabat (Maroc). Avant de commencer le cours des lentilles minces, les élèves du groupe expérimental et témoin, ont été invités à répondre au l'administrateur du Pré-test, la durée du questionnaire a duré 20 minutes. Le questionnaire est conçu en langue arabe (langue d'enseignement de matières scientifiques). Les réponses des élèves ont été analysées qualitativement, afin d'identifier les conceptions erronées des élèves (avant et après l'enseignement). Cette identification, nous aide à faire la conception d'une activité d'apprentissage dans le but d'aider les élèves à évoluer leurs conceptions erronées en conceptions scientifiques. Le groupe témoin, a suivi le cours de manière classique (traditionnel) ; sans intervention. Par contre, pour le groupe expérimental, nous avons conçus une activité d'apprentissage basée sur le logiciel OptGeo. Dans un premier temps, nous avons distribué les élèves en 11 groupes (le groupe est composé de deux élèves), chaque groupe dispose un ordinateur portable sur lequel, le logiciel a été installé. Ainsi, une feuille de prédiction a été distribuée aux groupes dans le but de prédire ce qui passera dans les différents cas mentionnés sur la feuille de prédiction, avant de refaire le même travail sur l'ordinateur. Puis, dans un deuxième temps, nous avons demandé aux différents groupes de refaire le même travail sur le logiciel OptGeo (installé sur l'ordinateur) et, de constater ce qui se passera, en expliquant les différences entre ce qu'ils ont fait sur les feuilles de prédictions et ce qui était réalisée sur l'ordinateurs; s'il existe. En fin, nous avons invité les élèves du groupe expérimental de répondre à l'administrateur (posttest), pour faire une analyse quantitative et qualitative des résultats obtenus. Une analyse statistique a été menée à l'aide du logiciel SPSS 22.0.

\section{RESULTATS}

Le tableau 1 recense les conceptions des élèves sur les différents points traités par le questionnaire. Une analyse de l'administrateur (prétest et posttest) a été menée. Cette analyse qualitative a indiqué que les élèves ont de nombreuses conceptions erronées, sur la formation d'image et le processus de la vision. Le tableau 1 présente les conceptions erronées les plus fréquentes qui ont été trouvées chez les élèves. Nous avons constaté que les élèves, entre outre, ont développé de nouvelles conceptions erronées, même après la séance d'enseignement de l'optique géométrique ; d'autres ont développé des conceptions hybrides. Cependant, quelques conceptions erronées ont été apparu dans l'administrateur pré-test ont disparu dans l'administrateur posttest. 
Tableau 1. Les conceptions erronées trouvées avant l'apprentissage.

1. Comment l'image se forme à travers une lentille convergente?

\begin{tabular}{|c|c|c|}
\hline \multirow[t]{2}{*}{ Réponses } & \multicolumn{2}{|c|}{ Pré-test } \\
\hline & Fréquence & Pourcentage \\
\hline$\nabla^{\circ}$ & 25 & 48,07 \\
\hline & 14 & 26,09 \\
\hline$\theta \leqslant=8$ & 4 & 7,69 \\
\hline Autres & 9 & 17,307 \\
\hline 2. Qu'arrivera-t-il à l'image si nous cachons la moitié s & rieure de la 1 & un carton? \\
\hline Les rayons lumineux ne convergent pas & 15 & 15 \\
\hline Seulement la moitié de l'image qui apparait & 12 & 12 \\
\hline Aucune image ne sera apparue & 20 & 38,46 \\
\hline Autres & 5 & 9,61 \\
\hline 3. Comment l'œil de l'observateur voit l'image formé à & vers une lent & rente? \\
\hline $\begin{array}{l}\text { Les rayons lumineux arrivent dans les yeux et l'œil de } \\
\text { l'observateur voit l'image }\end{array}$ & 18 & 43,6 \\
\hline $\begin{array}{l}\text { l'observateur doit placer son œil dans à l'objet pour } \\
\text { puisse voir l'image }\end{array}$ & 20 & 38,46 \\
\hline Autres & 14 & 26,92 \\
\hline 4. si nous enlevons la lentille, que se passe-t-il sur l'écr & & \\
\hline Une image inversée sera formé & 15 & 28,8 \\
\hline L'image sera floue & 20 & 38,46 \\
\hline L'image sera claire, mais sans inversement & 10 & 19,23 \\
\hline Autres & 7 & 13,46 \\
\hline 5. Qui se passera, si nous cachons l'objet lumineux par & carton? & \\
\hline $\begin{array}{l}\text { La lumière émanant de l'objet lumineux se propage } \\
\text { dans la direction où la lentille se trouve. }\end{array}$ & 16 & 30,76 \\
\hline L'image sera apparaître à l'envers & 17 & 32,69 \\
\hline L'image sera floue & 11 & 21,15 \\
\hline
\end{tabular}

Ce tableau montre que les élèves ont des conceptions diffèrent, les connaissances scientifiques. Ces conceptions ont été évoluées, comme le montre tableau 2, nous présenterons les conceptions les plus fréquentes qui ont été trouvé chez les élèves après une séance d'enseignement. 
Tableau 2. Les conceptions des élèves qui ont été trouvées. Après l'apprentissage. 1. Comment l'image se forme à travers une lentille convergente?

\begin{tabular}{|c|c|c|c|c|}
\hline \multirow[t]{2}{*}{ Réponses } & \multicolumn{2}{|c|}{ Posttest(témoin) } & \multicolumn{2}{|c|}{ Posttest (experimental) } \\
\hline & Fréquence & $\%$ & Fréquence & $\%$ \\
\hline & 6 & 2 & 16 & 72,7 \\
\hline & 16 & 15,333 & 6 & 27,3 \\
\hline- & 5 & 16,666 & 0 & 0 \\
\hline Autres & 3 & 1 & & \\
\hline \multicolumn{5}{|c|}{ 2. Qu'arrivera-t-il à l'image si nous cachons la moitié supérieure de la lentille par un carton? } \\
\hline Les rayons lumineux ne convergent pas & 13 & 43,33 & 6 & 27,27 \\
\hline Seulement la moitié de l'image qui apparaît & 12 & 40 & 2 & 9,09 \\
\hline $\begin{array}{l}\text { L'image sera formé, même si la moitie } \\
\text { supérieur de la lentille est cachée }\end{array}$ & 2 & 6,666 & 14 & 63,63 \\
\hline Autres & 3 & 10 & 0 & 0 \\
\hline \multicolumn{5}{|c|}{ 5. Qui se passera si nous cachons l'objet lumineux par un carton? } \\
\hline $\begin{array}{l}\text { Les rayons lumineux traversent le carton, et } \\
\text { l'image sera formée. }\end{array}$ & 3 & 10 & 0 & 0 \\
\hline L'image n'apparaîtra pas & 15 & 50 & 2 & 9,090 \\
\hline $\begin{array}{l}\text { Le carton est un milieu opaque, les rayons } \\
\text { lumineux ne peuvent pas traverser le cartons ; } \\
\text { aucun image sera formée }\end{array}$ & 10 & 33,333 & 18 & 81,81 \\
\hline Autres & 2 & 6,666 & 0 & 0 \\
\hline
\end{tabular}

Les résultats montrent que le groupe expérimental a performé mieux après l'intervention (activité d'apprentissage informatisée). Les élèves commissent de conceptions erronées (avec un faible pourcentage $3 \%$ ) et, plus $26 \%$ des conceptions hybrides, et plus de $70 \%$ des conceptions qui sont plus scientifiques; dans le posttest. Le tableau 3 résume les statistiques descriptives des scores au questionnaire entre les prétests et les posttests pour les deux groupe (expérimental et témoin). L'analyse statistique a montré que le moyenne du groupe témoin (après l'apprentissage) est 6,5278 tandis que pour le groupe expérimental est 13,3158. 
Tableau 3 : Résultats des statistiques descriptives.

\begin{tabular}{|l|l|l|l|l|l|}
\hline \multicolumn{2}{|l|}{} & \multicolumn{2}{c|}{ Prétest } & \multicolumn{2}{c|}{ Posttest } \\
\hline Groupe & $\mathrm{N}$ & Moyenne & Ecart-type & Moyenne & Ecart type \\
\hline Témoin & 24 & 2,9861 & 1,28691 & 8,0417 & 1,988 \\
\hline Expérimental & 23 & 3,6842 & 1,20386 & 13,8696 & 2,9188 \\
\hline
\end{tabular}

Le tableau3 montre les résultats du T-test pour l'administrateur pré-test et posttest pour les deux groupes (expérimental et témoin).

Tableau 4 : Résultats du T-test.

\begin{tabular}{|l|c|c|}
\hline & Pré-test & Posttest \\
\hline Témoin & 2,9861 & 8,0417 \\
\hline Expérimental & 3,6842 & 13,8696 \\
\hline T-test & 2,025 & 15,173 \\
\hline Sig. (bilatérale) & 0,05 &, 000 \\
\hline
\end{tabular}

Les résultats mentionnées ci-dessus, montrent que même si aucune différence significative n'existait avant l'enseignement, entre le groupe expérimental et le groupe témoin $(p=0.05)$. Le groupe expérimental a obtenu significativement plus de gains conceptuels $(p=0,000)$ après la réalisation de la situation d'apprentissage basée sur les nouvelles technologies (logiciel de simulation OptGeo). Ces résultats nous permettent de dire que la compréhension conceptuelle des élèves du groupe expérimental est meilleure que celle des élèves du groupe qui ont suivi le cours de manière classique.

\section{DISCUSSION}

L'objectif principal de cette recherche, c'était de révéler les conceptions erronées chez les élèves marocains de la deuxième du secondaire collégial, sur la formation d'image et la vision, le comportement de la lumière. Pour aborder la première question de notre recherche, 16 conceptions erronées ont été identifiées à travers les réponses des élèves dans l'administrateur du pré-test. Ces conceptions erronées ont été répertoriées dans le tableau 1. Ainsi, le pourcentage de ces conceptions erronées dans les deux groupes (expérimental et témoin) et leur fréquence. Les élèves ont des perceptions que « la lumière se déplace vers les yeux, puis la lumière est réfléchie vers un objet, de sorte que l'objet puisse être vu ». Certains élèves, n'ont pas pu fournir une explication claire et scientifique sur le processus de vue. Ils ont négligé la lumière réfléchie par la lentille et qui atteignent les yeux. Ces conceptions erronées (ou idées fausses) ont été également révélées dans de nombreuses études à l'échelle international [1,2]. En outre, [16,17] ont affirmé que les élèves ont des conceptions erronées sur le processus de vision des objets. Les élèves ont pensé que la vision se fait par un rayon lumineux émanant de l'œil vers l'objet. Malgré, que Les élèves connaissent l'expérience de 
la vue et ses conditions nécessaires comme, la possession des yeux ouverts, l'existence d'objet visible avec un éclairage suffisant. Malgré tout, l'explication théorique de la vision n'est, en revanche, ni évidente, ni bien connue chez les élèves [17]. Nous avons demandé aux élèves de préciser l'emplacement de l'œil de l'observateur, pour qu'il puisse voir l'image formée à travers la lentille convergente, ils ont tracé le schéma suivant :

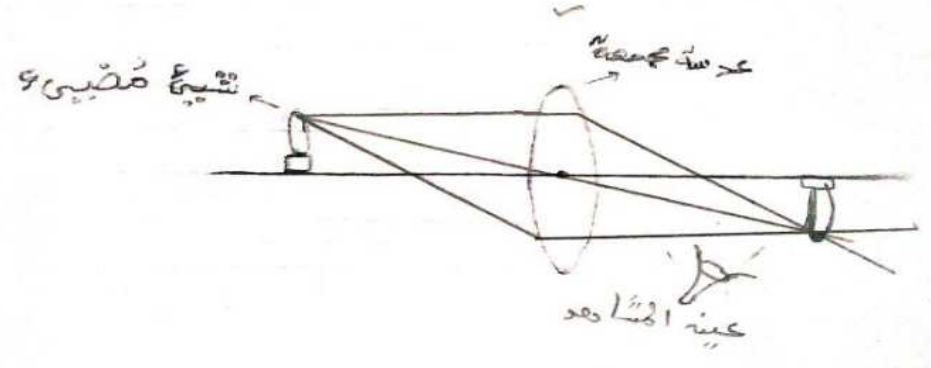

Figure .1 l'emplacement de l'œil de l'observateur, tracé par les élèves.

Apres l'intervention, nous avons demandé aux élèves de répondre à même question, avec une explication. Voir schéma.2

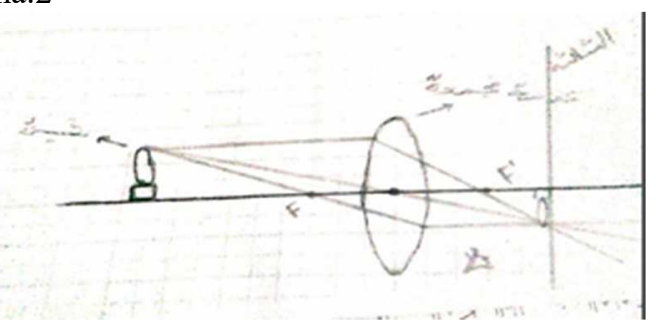

Figure .2 l'emplacement de l'œil de l'observateur, tracé par les élèves

De plus, les résultats montrent que les élèves ont des conceptions erronées sur le processus de formation d'image à travers une lentille convergente. Ils pensaient, entre outre, que « le rôle de la lentille c'est inverser l'image et non de la former », «la présence de l'écran est nécessaire pour que l'image sera formée », «si un rayon traverse la lentille ne change pas son direction ». Ceci est similaire aux résultats de quelques recherches $[1,2,18]$. De plus, nous avons constaté comme le montre schéma 1 que les élèves ont fait un passage de conception holistique "l'image voyageuse" à une "conception hybride" vers des conceptions plus scientifiques, ceci est similaire avec les recherches qui ont été menées par $[1,2]$. Les élèves n'ont pu déterminer le bon positionnement de l'œil, pour que l'observateur voit l'image de l'objet lumineux.

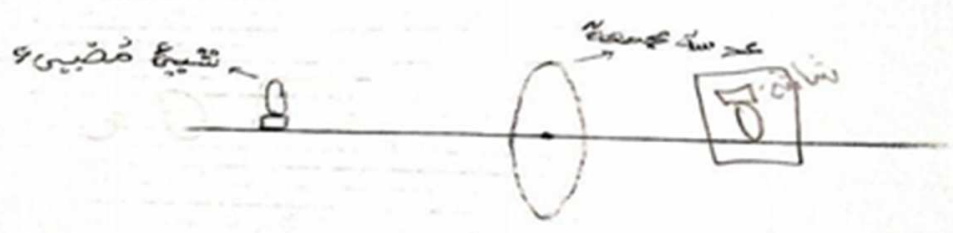



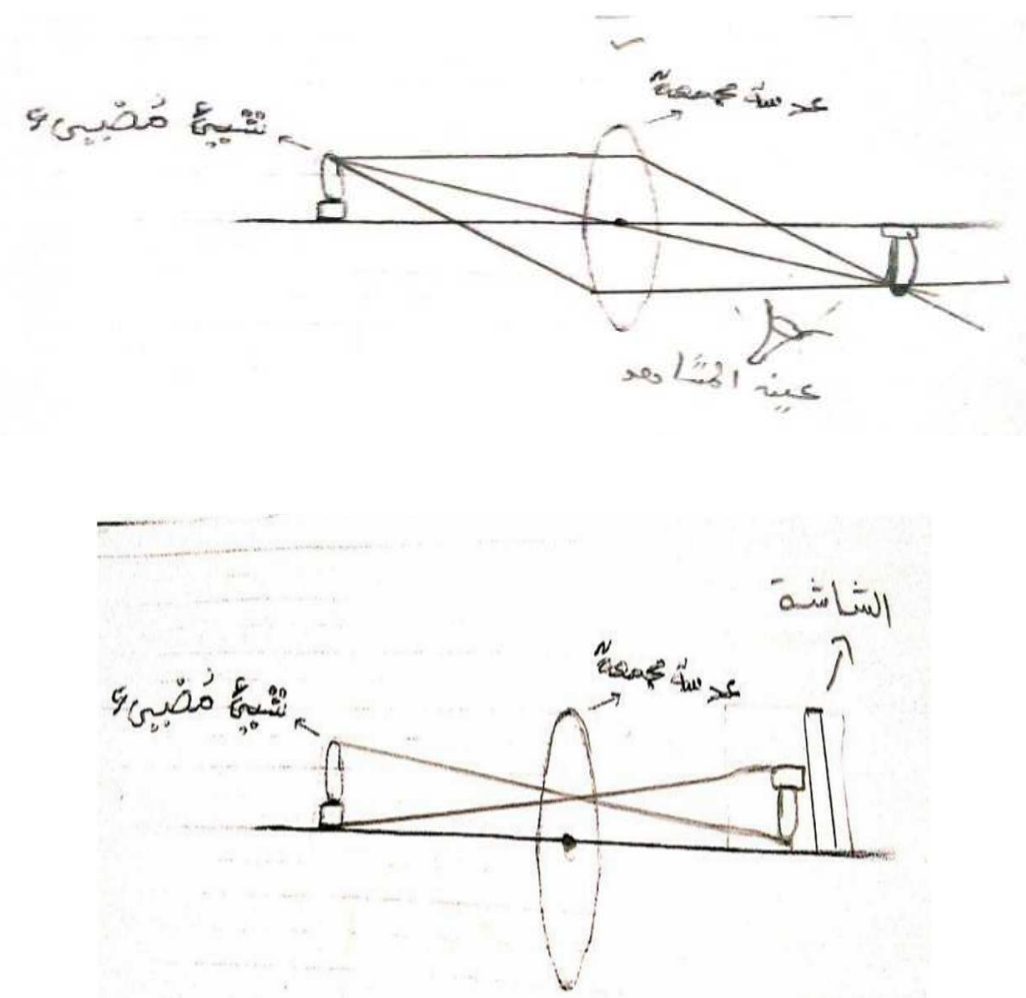

Figure $\mathbf{3}$ Conceptions des élèves à propos de Formation d'Image.

Il est clair que les élèves de la deuxième année du secondaire collégial ne comprennent pas très bien le comportement de la lumière. Ils croient, que nous cachons l'objet lumineux, les rayons lumineux traversent le carton, «si nous cachons l'objet lumineux l'image sera formée ». Ensuite, nous résultats dans le tableau 1, montrent que les conceptions erronées des élèves sur la formation d'image et la vision sont très résistants à l'enseignement traditionnel $[8,19]$; seuls quelques élèves ont réussi à changer ces conceptions erronées, notamment du groupe témoin.

Une approche quantitative a été utilisée à la deuxième question de notre recherche. Le moyen du groupe expérimental est passé de 3,6 en pré-test à 13,8696 en posttest. Même s'il $\mathrm{y}$ avait des différences statistiquement significatives entre la moyenne scores du groupe expérimental dans le pré-test et posttest en faveur le posttest $(t=15,173 ; p=0,000)$.

\section{Conclusion}

De nombreuses recherches dans le monde ont été menées, sur les conceptions erronées des élèves. Les conclusions de ces recherches ont montré que les élèves ont des conceptions erronées et, que ces conceptions ont une influence sur l'acquisition des connaissances scientifiques. Pour cette raison, il est nécessaire de faire des recherches sur les conceptions préalables des élèves avant d'entamer l'enseignement et d'appliquer une stratégie d'apprentissage qui favorise le changement conceptuel et de mettre les élèves dans une situations d'apprentissage qui mène les élèves à faire un conflit cognitif, dans le but de remplacer les conceptions erronées des élèves par des conceptions plus scientifiques. Cette recherche a confirmé que les élèves marocains de la deuxième année du secondaire collégial ont de nombreuses conceptions erronées sur, la formation d'image, la vision d'image et le 
mécanisme de la vision. De plus, certains de ces conceptions sont conformes avec les conceptions qui ont été identifiés par les chercheures à l'échelle internationale.

\section{Références}

1. I.Galili,Students'conceptualchange in geometrical optics.INT.J.SCI.EDUC,18,847868(1996).

2. I.Galili, S.Bendal, F.Goldberg, The effects of prior knowledge and Instruction on Understanding Image Formation. Res.In.Sci.Tea,30,1169-1187(1993).

3. I. Galili, A.Hazan, Learners' knowledge in optics: interpretation, structure and analysis. INT.J.SCI.EDUC, 22, 57- 88(2000).

4. L.Viennot, W.Kaminski,Can we evaluate the impact of a critical detail ? the role of a Type of Diagram in Understanding Optical Imaging.INT.J.SCI.EDUC, 28, 18671885(2006).

5. A.FAWAZ, L. Viennot, image optique et vision : Enquête en classe de première au liban.Bull.Union.Phy, 686,1125-1146(1985).

6. D.ARNANTONAKI, un modèle précurseur sur la lumière pour les élèves de 10 à 11 an : cadres théoriques ET méthodologiques. Educ.J.Uni.Patras UNESCO Chair,3,7483(2016).

7. H.Eshach, M.N.Fried, should science be taugh in early childhood.J.Sci. Edu.Tech, 14,316-336(2005).

8. P.Potvin, Faire apprendre les sciences et la technologie à l'école, Epistémologie, didactique, sciences cognitives et neurosciences au service de l'enseignement. (Presse de l'université Laval, Québec, (2018).

9. M.Papandreou, M.Trezi, Exploring children's ideas about natural phenomena in kindergarten classes: designing and evaluating 'eliciting activities. Review of science, Mathematics and ICT EDUCATION .2,27-47(2011).

10. J.M.Boilevin, Rénovation de l'enseignement des sciences physiques et formation des enseignants.279 (Bruxelles : De Boeck, 2013).

11. F.Cordier, A.Tiberghien.le discours dans A.Tiberghien (dir), Des connaissances naïves au savoir scientifique.2-9(CNRS.Unive.Lum Lyon, 2003).

12. M.Droui, L'impact d'une simulation sur des dispositifs mobiles et en situation de collaboration sur la compréhension de l'effet photoélectrique au niveau collégial. (Thèse de Doctorat, Université Montréal, ,2013).

13. J.K.Gilbert, M.Watts, R.J.Osborne, Students' conceptions .Phys.Educ, 17, 62-66 (1982)

14. A. Dumas Carré, A.Weil-Barais, k.Ravanis, F.shourcheh, interactions maitre-élèves en cours d'activités scientifiques à l'école maternelle: approche comparative.Bulle.Bulletin psychologie, 56, 494-508 (2003).

15. C.Siry and I.Kremer,Children Explain the Rainbow: Using Young Children's Ideas to Guide Science Curricula.J.S.E.C.T, 20,643-655(2011).

16. G.A.Winer,J.E.,Cottrell,V.Gregg,J.S.Fournier,L.A.Bica,Fun-damentally misunserstanding visual perception: Adults'belief in visual per-ception: Adults' belief in visual emissions.Americ.Psyco, 75 ,417-424(2002).

17. N. J. Selley, Children's ideas on light and vision. INT. j. sci. EDUC.18,713-723(1996) 
18. M.John, J.Maisha Molepo,M.Chirwa, South African Learners' Conceptual Understanding about Image Formation by Lenses.EURASIA Journal of Mathematics Science and Technology Education. 6 ,1723-1736(2017).

19. F.M. Goldberg,L.C. McDermott, An investigation of student understanding of the real image formed by a converging lens or concave mirror.Am.J.Phys.55,108-119(1987).

20. A. Boustan sarığlan, H.Küçüközer, Comparison of high school students' ideas about momentum and Impulse conceptions before and after instruction. Procedia - social and behavioral sciences, 116, 3771 - 3775 (2014). 